\title{
Transnasal Endoscopic Repair of Cerebrospinal Fluid Rhinorrhea
}

\author{
${ }^{1}$ Devang P Gupta, ${ }^{2}$ SK Dinesh, ${ }^{3}$ Ashil D Manavadaria
}

\section{ABSTRACT}

Introduction: Cerebrospinal fluid (CSF) leak can arise as a complication of trauma, hydrocephalus, endoscopic sinus surgery, or it may occur spontaneously without any identifiable cause. Surgical repair is recommended in patients who do not respond to the conservative management. In recent years, transnasal endoscopic approach has become the preferred method for repairing CSF leaks, and better outcomes have been reported as compared with the intracranial approaches that were previously used. The objective of this study was to analyze the outcome of transnasal endoscopic repair of CSF rhinorrhea.

Materials and methods: This prospective study was conducted in the Department of ENT, B.J. Medical College, Ahmedabad, India, from April 2013 to July 2016. Twenty-five patients with CSF rhinorrhea were included in the study. They were diagnosed based on the clinical evaluation, computed tomography, and magnetic resonance imaging. These patients did not respond to conservative management and were operated transnasally using rigid endoscope. Patients were followed up for a mean duration of 9 months, and the outcome was analyzed.

Results: The patients included in the study ranged in the age group of 12 to 55 years. Among the patients, 15 were females and 10 were males. The cause of CSF rhinorrhea was traumatic in 18, idiopathic or spontaneous in 7. In 12 patients, the site of leak was cribriform plate, 6 from ethmoid, 5 from sphenoid sinus, and in 2 frontal sinuses were affected. Primary surgery was successful in 23 of cases. In two cases, reexploration had to be performed. Overall success rate was $100 \%$.

Conclusion: Transnasal endoscopic repair of CSF rhinorrhea is highly successful, safe, and less traumatic.

Keywords: Cerebrospinal fluid, Endoscopic repair, Transnasal.

How to cite this article: Gupta DP, Dinesh SK, Manavadaria AD. Transnasal Endoscopic Repair of Cerebrospinal Fluid Rhinorrhea. Clin Rhinol An Int J 2017;10(2):45-48.

\section{Source of support: Nil}

Conflict of interest: None

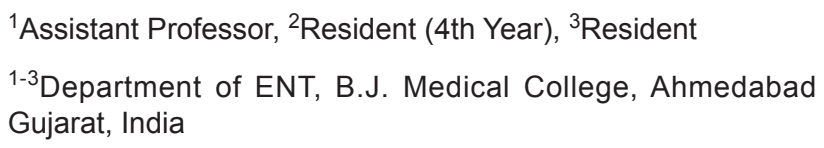

Corresponding Author: Devang P Gupta, Assistant Professor Department of ENT, B.J. Medical College, Ahmedabad, Gujarat India, Phone: +919824064313, e-mail: sapna_gupta76@ yahoo.com

\section{INTRODUCTION}

Cerebrospinal fluid (CSF) rhinorrhea is the leakage of CSF from subarachnoid space into the nasal cavity due to defect in the dura, bone, and mucosa. Cerebrospinal fluid leaks of anterior skull base present one of the more difficult challenges in endonasal endoscopic surgery, concerning an area, i.e., anatomically complicated and technically demanding to access. The challenge recreated a barrier between cranial vault and the nasal cavity to prevent and eliminate CSF leaks and protect the brain from exposure to secondary infection. Etiology varies from trauma, iatrogenic injuries, congenital malformations, tumor, or idiopathic. Etiology affects the risk of recurrence and thus the method of repair by having an impact on the defect size, location, degree of dural involvement, the likelihood of elevated intracranial pressure (ICP), and the possibility of meningoencephalocele protrusion. ${ }^{1}$ Risk of meningitis from untreated CSF rhinorrhea ranges from $10 \%$ annually up to $40 \%$ in long-term follow-up. The approach can vary from intracranial approaches that were first described by Dandy. ${ }^{2}$ The morbidity of intracranial approaches may include hemorrhage, cerebral edema, anosmia, memory deficit, and osteomyelitis of bone flap. Wigand ${ }^{3}$ reported the first endoscopic repair of CSF fistula. This study determines various causes and outcome of endoscopic repair of CSF leak. A meta-analysis showed a success rate of $90 \%$ in the first attempt and $97 \%$ overall with the endoscopic technique. ${ }^{4}$

\section{AIMS AND OBJECTIVES}

- To evaluate the outcome of endoscopic repair of CSF rhinorrhea.

- To study the techniques adopted for endoscopic repair and various graft materials used.

\section{MATERIALS AND METHODS}

Retrospective study was done on 25 patients treated for CSF leaks of the anterior skull base presenting to our department of ENT, Civil Hospital, Ahmedabad, India, between April 2013 and July 2016. Data were collected according to the patient's characteristics, CSF fistula, surgical techniques, materials used to repair the defect, and complications. 


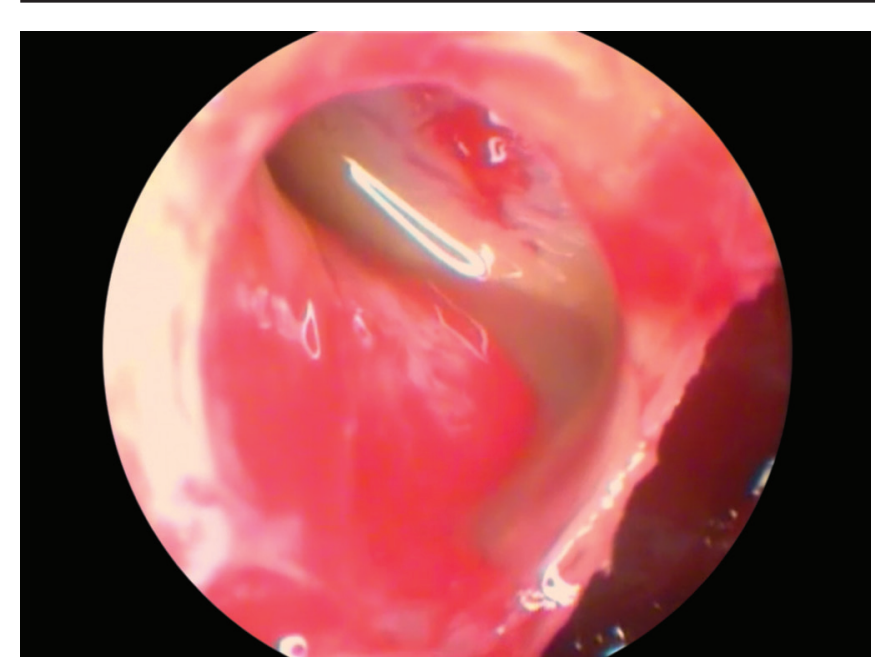

Fig. 1: Fluorescein dye leak from lateral wall and roof of sphenoid

\section{Inclusion Criteria}

- Defects less than $1.5 \mathrm{~cm}$ in size

- Defects of cribriform, ethmoid, sphenoid, and frontal recess area

\section{Exclusion Criteria}

- Defects greater than $1.5 \mathrm{~cm}$

- Defect of posterior wall of frontal sinus

- Multiple skull base fractures

- Tumors with increased ICP

\section{TREATMENT}

Conservative measures were used in critical cases that included strict bed rest, avoiding strain, head end elevation, laxatives, antitussives, oral diuretics: Acetazolamide, lumbar drain (r), and prophylactic antibiotics. Surgical steps include intrathecal dye (fluorescein dye) which was injected through lumbar puncture $0.25 \mathrm{~mL}$ of $5 \%$ fluorescein in few cases preoperatively. On endoscopy, CSF leak appeared to be bright yellow green colored.

\section{ENDOSCOPIC REPAIR}

Exposure of flaw was by complete ethmoidectomy/ maxillary sinusotomy/frontal sinusotomy/sphenoidotomy/ middle/superior turbinectomy as required. The defect site surrounded by mucosa, about $5 \mathrm{~mm}$, was stripped away. Encephaloceles were reduced by bipolar electrocautery at the stalk. Graft was placed followed by fibrin glue. In all cases, we used fibrin glue to improve adherence of the graft, which was supported in place with layers of surgicel to separate the graft from the packing material, to prevent avulsion of the graft or flap during its removal.

Absorbable nasal packing was placed adjacent to the grafts followed by nonabsorbable packing. Graft

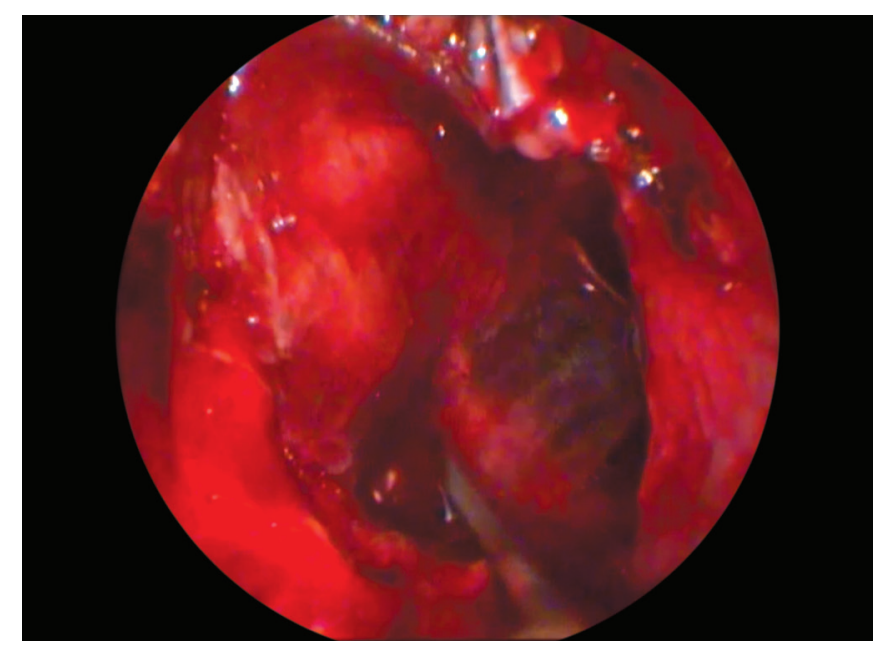

Fig. 2: Tensor fascia lata graft kept over lateral wall of sphenoid sinus, overlay technique

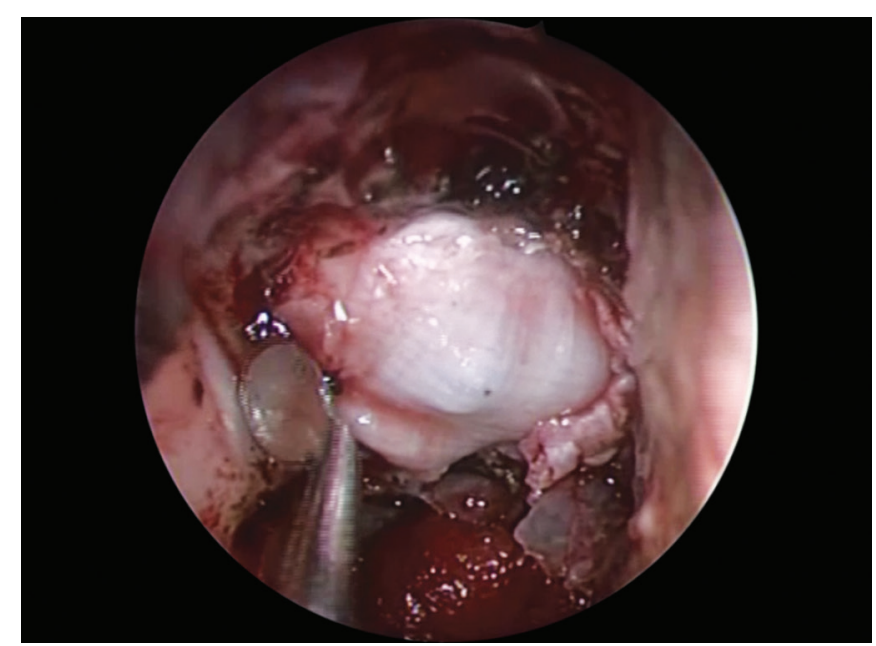

Fig. 3: Underlay technique in meningoencephalocele repair

materials used for repair were fat, fascia lata, nasal septal mucoperichondrial flap/Hadad flap, and septal cartilage. Techniques used for graft placement are (Figs 1 to 3):

- Overlay technique when size of defect is $<3 \mathrm{~mm}$

- Underlay technique when size of defect is $>3 \mathrm{~mm}$

- Bath plug technique when size of defect is up to $1.5 \mathrm{~cm}$.

\section{Postoperative Management}

Patients were advised bed rest for 48 to 72 hours, with the head raised up $30^{\circ}$ to $45^{\circ}$. Nonabsorbable nasal packing was removed within 3 to 4 days. Stool softeners, antihistamines, and antiemetic were administered as needed to reduce intraabdominal pressure for 2 weeks. IV antibiotics were used for 5 days followed by oral antibiotics for 2 to 3 weeks. Patients were instructed to avoid strenuous activity. Mean duration of follow-up was 9 months (range 4 months to 2 years). 


\section{DIAGNOSIS}

Accurate identification of the CSF leakage site is essential for a successful surgical repair. In some cases, the diagnosis through nasal endoscopy can give an exact finding. However the dimensions, bony anatomy of the surrounding area cannot be assessed on simple diagnostic nasal endoscopy. Leaking of fluid from the nose if placed on absorbent filter paper may outcome in the double-ring sign, which is a central circle of blood and an outer ring of CSF. Beta-2 transferrin assay is more specific for CSF; however, in case of associated orbital injuries, this can be unreliable due to the presence of beta-2 transferrin in vitreous humor. Beta-2 transferrin is a carbohydrate-free (desialated) isoform of transferrin, which is almost exclusively found in the CSF. Beta-2 transferrin has a sensitivity of near $100 \%$ and a specificity of about $95 \%$. Glucose detection using Glucostix test strips is not recommended as a confirmatory test due to its lack of specificity and sensitivity. Injection involving a solution of 0.5 to $10 \%$ fluorescein dye is injected and the patient is then examined roughly from 30 minutes to an hour later with an endoscope. High-resolution, thin section axial and coronal cranial and facial computed tomography (CT) contains all of the paranasal sinuses and petrous temporal bones in the scans are helpful in defect localization. Use of less irritating water-soluble positive contrast media, such as metrizamide combined with CT scanning and suitable image reconstruction can be often useful in pinpointing leak location. The CT cisternography cannot be undertaken in the presence of meningitis so in such cases, magnetic resonance imaging (MRI) with CT is done.

\section{RESULTS}

From an etiological point of view, dural defects were classified into two groups: 18 cases were posttraumatic, while 7 cases were spontaneous CSF leaks (Table 1). The most frequent site of the anterior skull base defect was the cribriform plate, followed by the ethmoid and sphenoid sinus (Table 2). There was associated encephalocele in two cases. Closure technique was based on the size and

Table 1: Classification of dural leaks into two groups

\begin{tabular}{llll}
\hline Leak type & No. of patients & Gender & Recurrence \\
\hline Traumatic & 18 & $11 \mathrm{~F} / 7 \mathrm{M}$ & \\
Spontaneous & 7 & $4 \mathrm{~F} / 3 \mathrm{M}$ & $2 \mathrm{~F}$ \\
\hline
\end{tabular}

Table 2: Distribution of patients according to site of leak

\begin{tabular}{lll}
\hline Site of leak & No. of patients & Percentage \\
\hline Cribriform plate & 12 & 48 \\
Ethmoid & 6 & 24 \\
\hline
\end{tabular}

location of the fistula. The most commonly used graft was fat combined with fascia lata. Success rate was $92 \%$ at the first attempt; only in two patients $(8 \%)$ a second surgical procedure was required, and none of it was necessary to use a craniotomy for closure. Follow-up throughout ranged from 4 to 24 months. The radiological diagnosis by CT cisternography confirmed the CSF leaks and locating the defect in 25/25 (100\%) patients. No patients had any complications following endoscopic CSF leak repair except two recurrence.

\section{DISCUSSION}

In recent years, we have seen the establishment of the endoscopic sinus surgery as a technique of choice for closure of CSF leak, seen as a less invasive technique with less morbidity and mortality, excellent view of the surgical field, and a higher success rate (about 95\%), replacing the usual techniques, such as transcranial and extracranial interventions that had a success rate of $70 \%$ with significant morbidity (anosmia was permanent sequel). ${ }^{5}$ Cerebrospinal fluid leak from the anterior fossa and sphenoid bone carries significant morbidity when inadequately treated and with serious complications, such as meningitis, subdural empyema, and brain abscess. In spontaneous CSF leak and meningo or encephaloceles, the failure rate can vary between 25 and $87 \%$. Autologous materials, such as abdominal fat, bone, nasal septum mucosa, fascia lata, and muscle can be used for repair. The graft can be attached with fibrin glue, hemostatic sponges, or Vaseline gauzes. Suitable resection of the mucosa around the bone defect is a must for secure graft attachment. Graft can be positioned in an underlay or overlay form.

Factors, such as trauma, surgical, increased ICP, tumors, or other causes and the presence of congenital or acquired skull base defects predispose a patient to progress CSF leak; quiet a lot many cases are idiopathic or spontaneous. Dural defects are still rare, and must be closed because of the risk of developing meningitis or pneumocephalus. Detailed history and clinical examination can be increased by laboratory tests of rhinorrhea samples for B-2-transferrin detection or B-trace protein, which is a highly delicate technique. ${ }^{6}$ In our study, we mostly relied upon the high glucose content because these tests were not available. The CT scan picked up the skull base defect in $88 \%$ of cases; CT cisternography using intracranial metrizamide can be used when the site is not obvious on CT or endoscopy. The MRI T2 inversion recovery sequences with transposal time for fluid suppression fluid attenuated inversion recovery (FLAIR) is also more useful.

Fibrin glue portends to the final phase of the coagulation cascade, thrombin, and fibrinogen. The histopathological studies suggest that fibrin glue may 
cause an inflammatory response that may promote healing. However, the efficacy of fibrin glue in preventing CSF leaks remains controversial. In the first attempt, our series closure rate was $92 \%$, and $100 \%$ in the next attempt with fibrin glue. There was excellent correlation between the radiologic and the surgical findings. Lumbar drainage should be reserved for patients with higher ICP and the conservative measures that include bed rest, elevation of the head, and avoidance of straining activities, are sufficient to confirm the sealing of the leak. Combined use of fat and fascia lata is effective in CSF leak repair.

\section{CONCLUSION}

High-resolution CT scans and MRI are essential for preoperative planning and for locating sites of defects. For defects less than $1.5 \mathrm{~cm}$ in size of the cribriform, ethmoid, and sphenoid sinuses, endoscopic repair is the best option for a lasting repair. Compared with open approaches, endoscopic repair has a less morbidity, lower complication rate, and better overall success rate. Fluorescein may help in better visualization of these defects and seems to be a safe option if concentration is kept low. However, in defects less than $1.5 \mathrm{~cm}$ in size, and in unfavorable areas (posterior wall of frontal sinus, etc.), an open approach may still be required.

\section{REFERENCES}

1. Schlosser RJ, Wilensky EM, Grady MS, Bolger WE. Elevated intracranial pressures in spontaneous cerebrospinal fluid leaks. Am J Rhinol 2003 Jul-Aug;17(4):191-195.

2. Dandy WD. Pneumocephalus (intracranial pneumocele or aerocele). Arch Surg 1926 May;12(5):949-982.

3. Wigand ME. Transnasal ethmoidectomy under endoscopical control. Rhinology 1981 Mar;19(1):7-15.

4. Hegazy HM, Carrau RL, Snyderman CH, Kassam A, Zweig J Transnasal endoscopic repair of cerebrospinal fluid rhinorrhea: a meta-analysis. Laryngoscope 2000 Jul;110(7):1166-1172.

5. Senior BA, Jafri K, Benninger M. Safety and efficacy of endoscopic repair of CSF leaks and encephaloceles: a survey of the members of the American Rhinologic Society. Am J Rhinol 2001 Jan-Feb;15(1):21-25.

6. Woodworth BA, Prince A, Chiu AG, Cohen NA, Schlosser RJ, Bolger WE, Kennedy DW, Palmer JN. Spontaneous CSF leaks: a paradigm for definitive repair and management of intracranial hypertension. Otolaryngol Head Neck Surg 2008 Jun;138(6):715-720. 